\title{
GEOTACTICAL AND NEUROCHEMICAL PHENOTYPES OF DROSOPHILA MELANOGASTER FOLLOWING NIGELLA SATIVA EXPOSURE
}

\author{
Royhaan Folarin ${ }^{1 *}$, Kayode Ayodele ${ }^{1}$, Thomas Adenowo ${ }^{1}$, Muinat Adeyanju루 Joshua \\ Olugbode ${ }^{1}$, Esther Obadeyin ${ }^{1}$, \\ ${ }^{1}$ Neurophytotherapy Unit, Department of Anatomy, Olabisi Onabanjo University, Sagamu, Nigeria \\ ${ }^{2}$ Department of Biochemistry, Olabisi Onabanjo University, Sagamu, Nigeria \\ -*Corresponding Author: Royhaan Folarin \\ Neurophytotherapy Unit (NPTU), \\ Department of Anatomy, \\ Olabisi Onabanjo University, Sagamu, Nigeria \\ royhaan.folarin@oouagoiwoye.edu.ng \\ $+2347032064419$
}

\begin{abstract}
Drosophila melanogaster is a holometabolous frugivorous fly, with neurobiological and neurogenetic modelling importance owed to its small size, short life cycle, fast reproductive rate, low cost in maintenance and small tetra-chromosomal genome. Nigella sativa (Black seed) is a widely researched medicinal plant with numerous reported therapeutic activities in humans and rodents. Being the most abundant neurotransmitter in Drosophila, glutamate plays an important role in learning and memory, neuro-excitation but also neuro-inhibition. This research thus investigated the impacts of Nigella sativa on the survival rate, glutamate level and negative geotactical abilities in Harwich strains of Drosophila melanogaster. The experimental flies were exposed to varied concentrations of Nigella sativa oil for five days. The results showed a higher survival rate, glutamate level and negative geotactic ability for the lower dose flies, while the higher Nigella sativa dose flies recorded significantly lesser values in the trio. This indicates that Nigella sativa administered at $2.4 \mathrm{ml} / 4 \mathrm{ml}(60 \%)$ of feed may be lethal to the general survival and physiological functions of adult Drosophila. The lower dose however shows a high potential of improving locomotive and neurochemical activities in flies, as further studies are on to further identify the most therapeutic dose of Nigella sativa in Drosophila melanogaster, with a range suggested based on the findings of this research
\end{abstract}

Keywords: Drosophila melanogaster, Nigella sativa. Survival, Glutamate, Negative geotaxis 


\section{Introduction}

To identify alternative drugs for various human disorders, animal models that accurately recapitulate such disorder need to be developed. Drosophila melanogaster (Common fruit fly) is, on this account, rising in popularity, favoured by its low cost in maintenance, fast reproductive rate, small size, short life cycle, and tetra-chromosomal genome. It is therefore considered widely as a valid genetic model for several human disorders including Alzheimer's, Parkinson's, Huntington's, and spinocerebellar ataxia disorders, especially as over $65 \%$ of human diseaseassociated genes have a correlate (homolog) in Drosophila (Markstein, 2019).

Nigella sativa (also referred to as Habbatus-sawdaa - Black seed) is considered one of the most widely used medicinal plant across the world (Ahmad et al., 2013). While therapeutic potentials may be ascribed to many other medicinal plants available in nature, Nigella sativa is considered one of the greatest forms of therapeutics in the Tibbun-nabawiyy system of Islamic medicine, to such extent that it was declared a "remedy for all diseases except death" (Al-Bukhari, 1997). It is thus historically notable for its anti-inflammatory, analgesic, anti-pyretic, anti-bacterial, and anti-neoplastic potentials as proven by modern science (Ali \& Blunden, 2003; Naz, 2011).

Despite the extensive validations of biological activities and therapeutic potentials of Nigella sativa in humans and rodents, the phenotypes of Drosophila following Nigella sativa exposure has not been detailed in literature. To fill this gap in knowledge was thus the aim of this study.

Specifically, this research was aimed at determining the effect of Nigella sativa oil on the locomotory/geotactical and neurochemical properties of Drosophila melanogaster through negative geotaxis and glutamate assays in the flies. The study also assayed the survival rates of the flies upon dietary exposure to Nigella sativa oil.

\section{Materials and Methods}

\subsection{Acquisition and breeding of flies}

Male and female wild type Drosophila melanogaster (Harwich strains) were acquired from the Drug Metabolism and Molecular Toxicology Research Laboratory, Department of Biochemistry, University of Ibadan, Ibadan, Nigeria. The culture and feeding medium, which was largely cornmeal based, was hygienically adopted from protocols reported by Abolaji et al. (Abolaji et al., 2015). The flies were maintained under a cool and regulated temperature $\left(23 \pm 3^{\circ} \mathrm{C}\right), 60 \%$ 
humidity and natural day/night cycles in the Neurophytotherapy Lab (NPTL), Department of Anatomy, Olabisi Onabanjo University, Sagamu, Nigeria.

\subsection{Experimental Design and Dosing}

With each treatment vial containing 4 mLs of dispensed feed medium, the research experimental design consisted of three groups, namely Control, Low dose, and High dose. While the control group was maintained on the normal feed, the latter three groups were treated with 0.4 , and 2.4 mls of Nigella sativa oil respectively, corresponding to $10 \%$ and $60 \%$ of the feed volume respectively. The feed for each group was prepared separately while the respective volumes (or percentages) of Nigella sativa oil was added shortly after the cooked feed was done, before dispensing into the treatment vials. Each group consisted of four treatment vials each of which in turn had thirty flies of both genders, while the experiment lasted five (5) days.

\subsection{Negative geotaxis assay}

In order to assay for the negative geotactical effects of dietary exposure of the flies to Nigella sativa, ten flies from each treatment vial across the groups were introduced into the climbing apparatus made of calibrated vertical columns of high density polyethylene (length-15cm; diameter, $2 \mathrm{~cm}$ ) following anaesthesia on mild ice. An hour duration was allowed for the flies to completely recover from the anaesthetic exposure. A vertical distance of $8 \mathrm{~cm}$ above the bottom surface was marked with a circle around the circumferences of the column.

The flies were gently tapped to the bottom of the column, and the number of flies that passed the $8 \mathrm{~cm}$ mark by the $10^{\text {th }}$ second after the tapping were recorded as a percentage of total flies per trial. The assay was repeated for the same group five times, allowing for 1 minute rest period between each vial. The score for each trial was recorded as average of three, while the score for each group was recorded as an average of the four vials that constitute the group.

\subsection{Glutamate assay}

After 6 days of treatment with Nigella sativa, the flies from respective vials were anesthesised on mild ice and homogenized in $0.1 \mathrm{M}$ phosphate buffer, $\mathrm{pH} 7.0(1 \mathrm{mg}: 4 \mu \mathrm{L})$, and centrifuged at 5000 rpm for 5 minutes. The supernatant was thereafter decanted into labelled Eppendorf tubes for the determination of glutamate levels through spectrophotometry

\subsection{Statistical analysis}

Data were expressed as mean \pm standard error of mean. The experimental and control groups were compared using 1- way ANOVA and Bonferroni's Multiple Comparison Test. Ninety-five 
percent confidence interval was employed in determining statistical significance of group differences.

\subsection{Ethical Clearance}

This research was conducted with the approval of the ACUREC, Faculty of Basic Medical Sciences, Olabisi Onabanjo University, Sagamu, Nigeria.

\section{Results}

\subsection{Survival Rate}

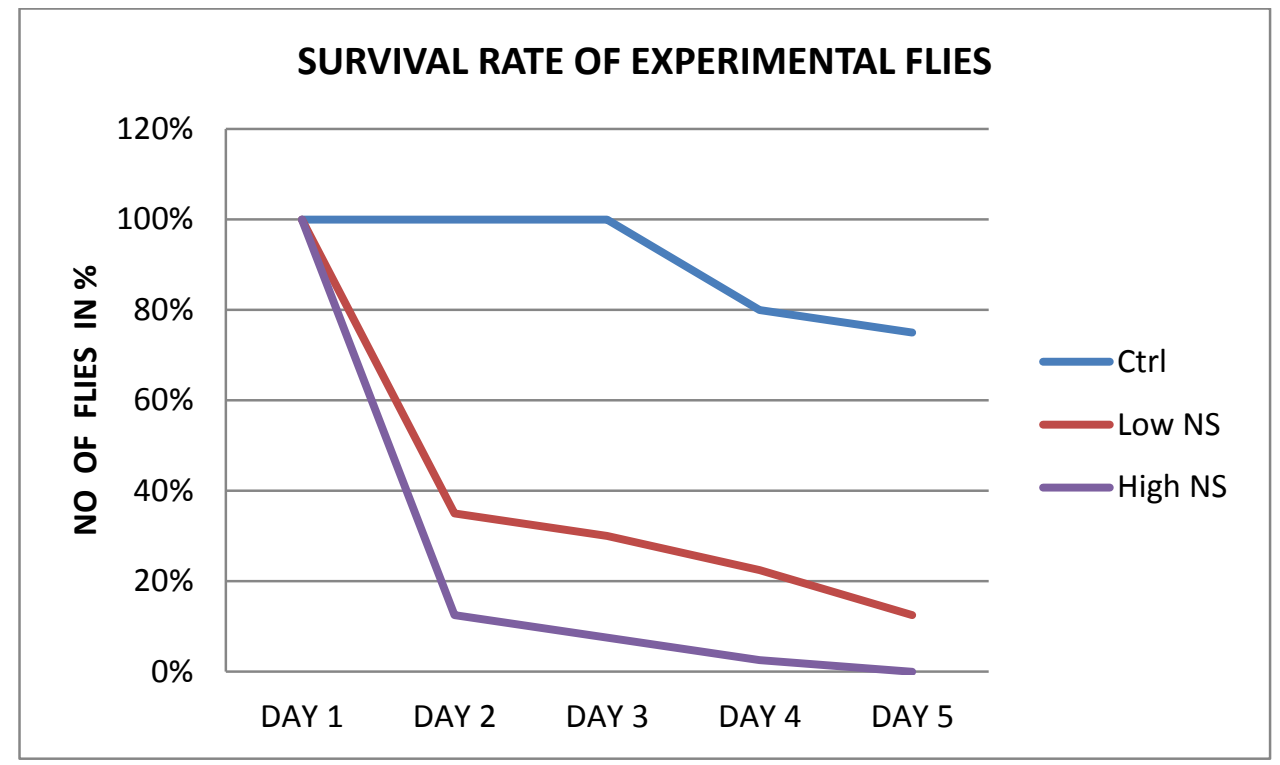

Figure 1: Curve showing the percentage mean $( \pm \mathrm{SEM})$ survival rates of the flies across the study duration

The table above illustrates the flies' survival rate from day one to day five of of the experiment.

A significant decline in the number of surviving experimental flies (Low and High NS) commenced from the second day of administration, falling to as low as $35 \%$ and $12.5 \%$ for low and high dose flies respectively, while no decline was recorded in the control flies until the fourth day of administration (falling to $80 \%$ ). Seventy-five percent (75\%) of the control flies eventually survived until the last day of the research, while the low dose groups had only $12.5 \%$ of the flies surviving through the last day of the research duration. The high dose flies however had no surviving fly by the end of the fifth day.

By the end of the last day (day 5), metamorphoses was observed in the high dose group, with a total of 9 adult Drosophila melanogaster recorded. 


\subsection{Negative Geotaxis Assay}

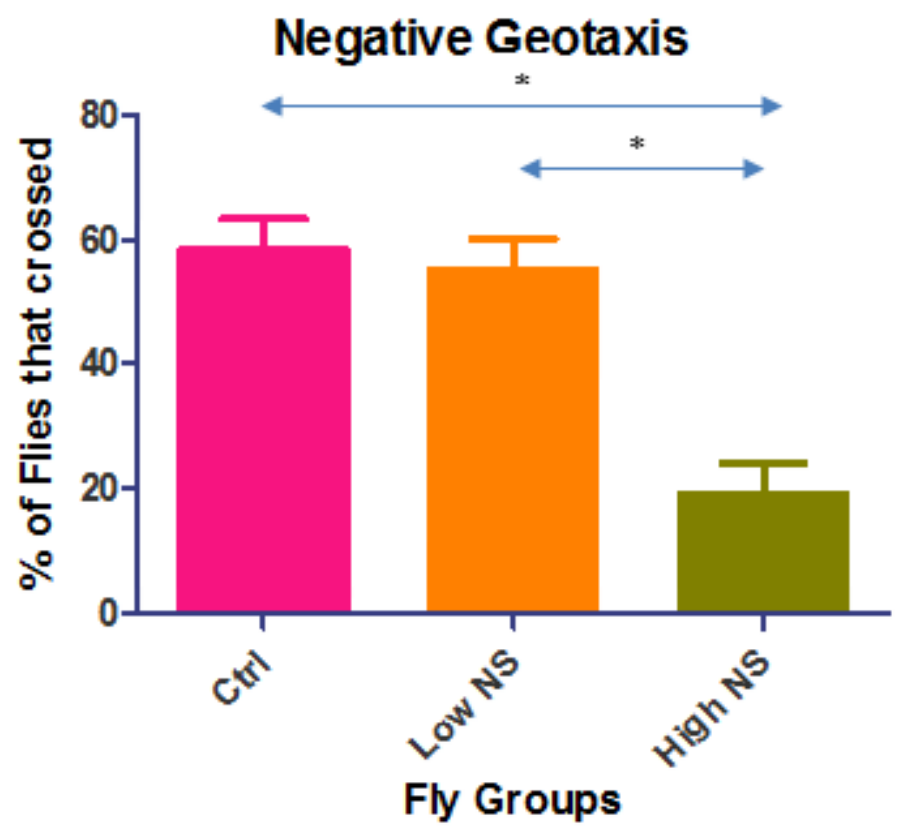

Figure 2 Graph showing the percentage mean $( \pm S E M)$ survival rates of the flies across the study duration $(*$ Significant with $95 \%$ Confidence interval)

The graph above shows the negative geotactical properties of the experimental and control flies across the groups. The control flies recorded the highest percentage of flies that crossed the denoted mark. The lower dose group (with 10\% Nigella sativa feed exposure) had the closest percentage of flies that crossed the mark to the control, with an insignificant difference of $3.3 \%$. The higher dose group ( $60 \%$ Nigella sativa) however had a significantly lower percentage of negatively geotactic flies than both the lower dose and the control. 


\subsection{Glutamate Assay}

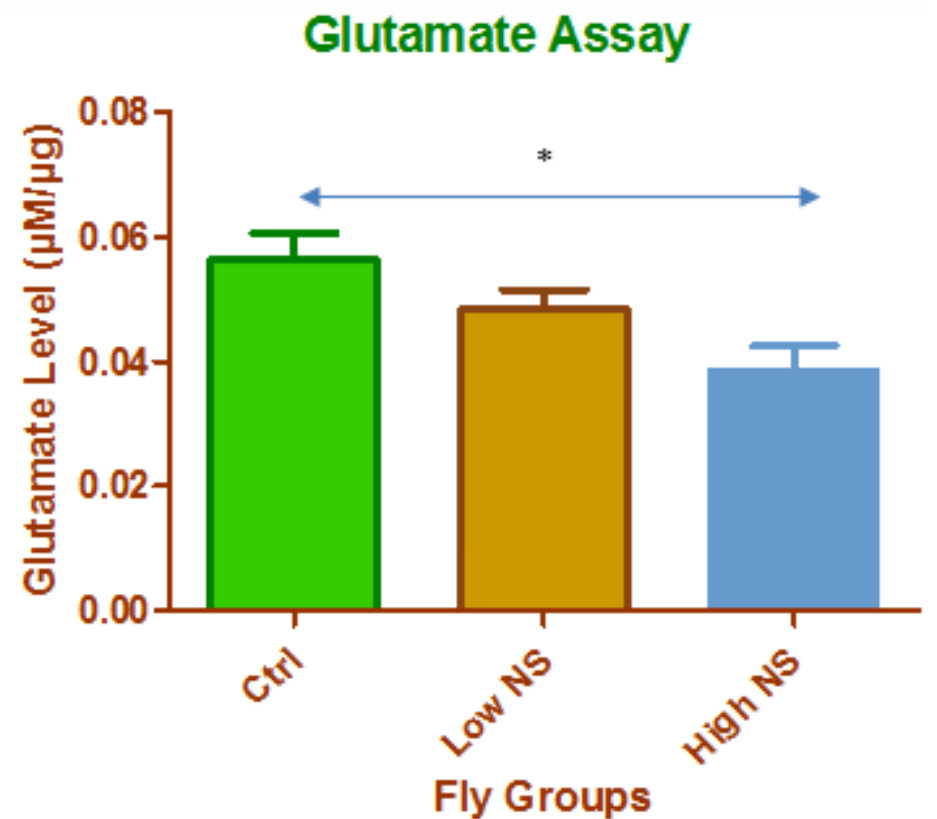

Figure 3 Graph showing the glutamate levels $\left( \pm\right.$ SEM) in the flies across the groups ${ }^{*}$ Significant with $\mathbf{9 5 \%}$ Confidence interval)

The glutamate levels in the flies were assayed using spectrophotometry, and the graph above illustrates a significant decrease in glutamate level of the high dose group when compared with the control. The low dose however maintained an insignificantly lower glutamate level than the control and insignificantly higher level than the high dose group. The glutamate levels upon further assessment was insignificantly higher in the living (and freshly sacrificed) flies in each respective group than in the previously dead flies.

\section{Discussion}

The impact of Nigella sativa on the life span of Drosophila was investigated through the survival assay, and the result presented above showed that $0.4 \mathrm{ml}$ of the oil per $4 \mathrm{ml}$ of feed $(10 \%)$ significantly reduced the life span of the flies by $87.5 \%$, while the higher dose $-2.4 \mathrm{ml} / \mathrm{ml}$ of feed $(60 \%)$ reduced the life span by a $100 \%$ within five days.

But despite the adverse results on the adult, the oil seemed not as lethal to the holometaboly of pre-adult stages of the flies as it was to the adults, for the Nigella sativa-containing media accommodated the metamorphosis of laid eggs into the various instars of larvae and subsequently adults. This may be explained by how able Drosophila larvae have been reported to move and survive in harsh conditions like hypoxia and anoxia, unlike adults which quickly get paralysed (Callier, Hand, Campbell, Biddulph, \& Harrison, 2015). The non-feeding third instar 
Drosophila larvae, among all stages, have also been reported as most resistant to gamma rays (Paithankar, Deeksha, \& Patil, 2017).

As part of its innate escape response, appropriate vertical movement is a critical phenomenon for the survival of flying animals (Bae et al., 2016). The locomotory and climbing activity of the flies was thus assayed after five days of Nigella sativa exposure at the described doses using the negative geotaxis assay.

Both experimental groups in this study showed Nigella sativa oil to effect a decrease in the percentage of negatively geotactic flies, with an inverse proportionality to the Nigella sativa dosage administered. A significantly impaired climbing behaviour was thus characterised to the high dose group compared to the control, but not the low dose group.

As Negative geotactic ability has been shown to be sensitive to several factors including oxidative stress, age, and previous cold exposure (Linderman, Chambers, Gupta, \& Schneider, 2012), high but not low dose of Nigella sativa has been shown through this study to adversely affect negative geotaxis in Drosophila.

Glutamate remains the most abundant excitatory neurotransmitter in Drosophila (Shin, Copeland, \& Venton, 2018), while glutamatergic neurons are also highly abundant in the Drosophila central nervous system (Liu \& Wilson, 2013). Neuro-excitability was thus assayed in the flies through glutamate neurotransmitter assay, and Nigella sativa has been shown to significantly decrease the glutamate level in the high but not the low dose group, suggesting lethality of the higher dose. The lower dose flies however maintained similar glutamate level with the control flies.

Neurotransmitters however may act on different spatial and temporal scales (Liu \& Wilson, 2013) and glutamate similarly has been reported to vary in its activities within Drosophila. It has been shown in some neuronal groups to promote wakefulness, increasing the duration of wake bouts through its excitatory role at neuromuscular junctions, while in others, it has been shown to promote sleep (Zimmerman et al., 2017). It has also been reported with inhibitory activities similar to GABA's in the antennal lobe (Liu \& Wilson, 2013), while its extracellular accumulation has been suggested to cause neuronal cell damage (Ikeda, Nakazawa, Abe, Kaneko, \& Yamatsu, 1989).

The above thus emphasise the need for further studies and characterisations to elucidate on which exact roles of glutamate is being mediated by Nigella sativa, in order to accomplish a better understanding and as well proffer evidenced hypotheses on the implications of its increase or decrease within the Drosophila homogenate. 
This study, after observing the expected reduction in glutamate release following death of the Drosophila flies, further reveals that it seems to take quite a while before the neurotransmitters are significantly lost in the dead flies. This was buttressed by the fact glutamate level recorded in the flies which previously died before the fifth day was only $20 \%$ lesser than that recorded in those sacrificed at the end of the fifth day. It may thus be important to determine the progressive rate of neurotransmitter loss for the purpose of knowledge.

\section{Conclusion}

Detailed above are novel findings reported in correlation of the impacts of Nigella sativa with neurochemical, behavioural and survival phenotypes of Drosophila melanogaster. The flies exposed to the higher dose of Nigella sativa showed the least level of negative geotactical locomotion, glutamate levels, and higher incidence of mortality in comparison with the control group. This suggests lethality of the higher dose to the general survival and physiological functions of Drosophila. But as the lower dose group showed milder and mostly insignificant differences from the control, this study indicates that Nigella sativa administered at low dose has a high potential of maintaining the locomotive and neuro-excitatory traits in Drosophila melanogaster if accurately administered.

The identification of the actual favourable and therapeutic dose of Nigella sativa oil in Drosophila flies thus remains a work in progress, and is hereby recommended within a range less than $10 \%$ of the feed medium for feed exposure, based on the findings reported above.

\section{Conflict of Interest}

The authors declare no conflict of interest.

\section{Acknowledgements}

The authors acknowledge and appreciate the valuable support and assistance from Dr Amos Abolaji and his wonderful team at the Drosophila Unit of the Drug Metabolism and Molecular Toxicology Research Laboratories, Department of Biochemistry, University of Ibadan, Ibadan, Nigeria, towards the acquisition and successful utilisation of the Drosophila stock employed for this study.

The lead author further shows immense gratitude to Prof. Marina Bentivoglio and Pof. Evelyne Sernagor of the IBRO/ISN Writing papers workshop, 2019, for their tutelage and guidance. Thank you. 


\section{References}

Abolaji, A. O., Kamdem, J. P., Lugokenski, T. H., Farombi, E. O., Souza, D. O., da Silva Loreto, É. L., \& Rocha, J. B. T. (2015). Ovotoxicants 4-vinylcyclohexene 1,2-monoepoxide and 4vinylcyclohexene diepoxide disrupt redox status and modify different electrophile sensitive target enzymes and genes in Drosophila melanogaster. Redox Biology, 5, 328339. https://doi.org/10.1016/j.redox.2015.06.001

Ahmad, A., Husain, A., Mujeeb, M., Khan, S. A., Najmi, A. K., Siddique, N. A., ... Anwar, F. (2013). A review on therapeutic potential of Nigella sativa: A miracle herb. Asian Pacific Journal of Tropical Biomedicine, 3(5), 337-352. https://doi.org/doi:10.1016/S2221$1691(13) 60075-1$

Al-Bukhari, M. (1997). The Translation of the Meanings of Sahih Al-Bukhari. Riyadh: Dar-usSalam Publications.

Ali, B. H., \& Blunden, G. (2003). Pharmacological and Toxicological Properties of Nigella sativa. Phytotherapy Research, 17(4), 299-305.

Bae, J.-E., Bang, S., Min, S., Lee, S.-H., Kwon, S.-H., Lee, Y., ... Chae, K.-S. (2016). Positive geotactic behaviors induced by geomagnetic field in Drosophila. Molecular Brain, 9(1). https://doi.org/10.1186/s13041-016-0235-1

Callier, V., Hand, S. C., Campbell, J. B., Biddulph, T., \& Harrison, J. F. (2015). Developmental changes in hypoxic exposure and responses to anoxia in Drosophila melanogaster. Journal of Experimental Biology, 218(18), 2927-2934.

https://doi.org/10.1242/jeb.125849

Ikeda, M., Nakazawa, T., Abe, K., Kaneko, T., \& Yamatsu, K. (1989). Extracellular accumulation of glutamate in the hippocampus induced by ischemia is not calcium dependent - In vitro and in vivo evidence. Neuroscience Letters, 96(2), 202-206.

https://doi.org/10.1016/0304-3940(89)90058-X 
Linderman, J. A., Chambers, M. C., Gupta, A. S., \& Schneider, D. S. (2012). Infection-Related Declines in Chill Coma Recovery and Negative Geotaxis in Drosophila melanogaster. PLoS ONE, 7(9), e41907. https://doi.org/10.1371/journal.pone.0041907

Liu, W. W., \& Wilson, R. I. (2013). Glutamate is an inhibitory neurotransmitter in the Drosophila olfactory system. Proceedings of the National Academy of Sciences, 110(25), 1029410299. https://doi.org/10.1073/pnas.1220560110

Markstein, M. (2019). Drosophila Workers Unite! (1st ed.). Retrieved from http://marksteinlab.org/wp-content/uploads/2019/01/MicheleMarksteinDrosophilaWorkersUnite-PREPRINT-JAN2019.pdf

Naz, H. (2011). Nigella Sativa: The miraculous herb. Pakistan Journal of Biochemistry and Molecular Biology, 44(1), 44-48.

Paithankar, J. G., Deeksha, K., \& Patil, R. K. (2017). Gamma radiation tolerance in different life stages of the fruit fly Drosophila melanogaster. International Journal of Radiation Biology, 93(4), 440-448. https://doi.org/10.1080/09553002.2016.1266056

Shin, M., Copeland, J. M., \& Venton, B. J. (2018). Drosophila as a Model System for Neurotransmitter Measurements. ACS Chemical Neuroscience, 9(8), 1872-1883. https://doi.org/10.1021/acschemneuro.7b00456

Zimmerman, J. E., Chan, M. T., Lenz, O. T., Keenan, B. T., Maislin, G., \& Pack, A. I. (2017). Glutamate Is a Wake-Active Neurotransmitter in Drosophila melanogaster. Sleep, 40(2). https://doi.org/10.1093/sleep/zsw046 